\section{O manejo não medicamentoso da hipertensão arterial sistêmica no Sul do Brasil}

\author{
Non-pharmacological management \\ of hypertension in Southern Brazil
}

\author{
1 Programa de Pós-graduação \\ em Saúde Coletiva \\ Universidade do Vale \\ do Rio dos Sinos, \\ São Leopoldo, Brasil. \\ 2 Faculdade de Medicina \\ Universidade Federal \\ de Pelotas, Pelotas, Brasil. \\ 3 Programa de Pós-graduação \\ em Epidemiologia, \\ Universidade Federal \\ de Pelotas, Pelotas, Brasil. \\ Correspondência \\ J. S. Dias-da-Costa \\ Departamento de Medicina \\ Social, Faculdade de \\ Medicina, Universidade \\ Federal de Pelotas. \\ Av. Duque de Caxias 250, \\ Pelotas, RS 96030 002, Brasil. \\ jcosta@epidemio-ufpel.org.br
}

\section{Abstract}

A cross-sectional population-based study was carried out among individuals aged 20 to 69 years in the urban area of Pelotas, Rio Grande do Sul, Brazil. The objective was to analyze characteristics of hypertensive patients in relation to management of arterial hypertension. Among the 1,968 interviewees, 380 were hypertensive (19.3\%), and $44.1 \%$ of these always consulted the same physician. Of the entire sample, $85.5 \%$ were sedentary, $48.2 \%$ showed excessive fat consumption, and 47.4\% smoked. Most physicians recommend the following: lowering salt consumption (98.4\%), cessation of alcohol consumption (93.7\%), for losing weight, diet and consumption of low-fat food (88.2\%), smoking cessation (73.2\%), and physical activity (68.9\%). The clinical management was classified as inadequate in 284 individuals (74.7\%). Increased probability of adequate management was found among: women, individuals $>50$ years, upper and middle-class patients, non-smokers, and patients with diabetes mellitus. Some findings showed positive aspects, but differences according to socioeconomic status evidenced lack of equity in care.

Hypertension; Risk Factors; Process Assessment (Health Care); Quality of Health Care

\author{
Giovani Sturmer 1 \\ Juvenal Soares Dias-da-Costa ${ }^{1,2}$ \\ Maria Teresa Anselmo Olinto 1 \\ Ana Maria Baptista Menezes 3 \\ Denise Petrucci Gigante ${ }^{3}$ \\ Silvia Macedo 2
}

\section{Introdução}

As doenças cardiovasculares integram o principal grupo de causas de morte no Brasil, representando $27,2 \%$ dos óbitos em 2002 1. No Rio Grande do Sul, em 2002, a mortalidade por doenças do aparelho circulatório foi a principal causa de óbitos, com $31,6 \%$ das mortes no Estado e $30,3 \%$ no Município de Pelotas 2.

A hipertensão arterial sistêmica é um dos principais fatores de morbidade e mortalidade nas doenças cardiovasculares 3 , apresentando uma elevada prevalência na população adulta no Rio Grande do Sul. Estudos epidemiológicos realizados em cidades do Estado têm revelado prevalências que variam entre 15 e 21,9\% 4,5, 6,7 . Recentemente, novo estudo transversal foi realizado com representação para todo o Estado do Rio Grande do Sul, os resultados mostraram 33\% de prevalência de hipertensão, confirmando a elevada freqüência da doença 8 .

Modificações no estilo de vida, especialmente a redução de peso, a diminuição da ingestão de sal e de álcool, como também a adoção de dieta hipocalórica, além da prática de atividade física regular, mostraram-se comprovadamente eficazes na redução da pressão arterial, na melhora da efetividade anti-hipertensiva e na diminuição do risco cardiovascular associado ${ }^{9}$. Entretanto, a incorporação efetiva dessas recomendações e estratégias de manejo na prática clínica ainda é desconhecida. 
Alguns autores têm recomendado a implantação de atividades sistemáticas de avaliação da assistência à saúde no Brasil 10,11,12, chegando a considerar que os indicadores de processo são de coleta mais fácil e mais rápidos, sendo mais sensíveis a mudanças 13,14. Assim, o objetivo do presente estudo foi avaliar as condições de manejo clínico não medicamentoso de indivíduos com hipertensão arterial sistêmica em Pelotas. O estudo procurou também caracterizar os indivíduos classificados como recebendo manejo inadequado para hipertensão arterial sistêmica.

\section{Metodologia}

Durante o período de dezembro de 1999 a abril de 2000, foi realizado um estudo transversal de base populacional com o objetivo de estudar diversos aspectos relacionados à saúde da população adulta, de 20 a 69 anos, residentes na zona urbana do Município de Pelotas.

Pelotas é um município situado ao sul do Estado do Rio Grande do Sul, tendo uma população estimada em 320 mil habitantes, em 2000. O Plano Municipal de Saúde descreveu uma extensa rede assistencial constituída por 48 unidades sanitárias, quatro ambulatórios de especialidades e cinco hospitais. A essa rede somamse inúmeros serviços contratados. O estágio de municipalização da saúde está implantado na forma de gestão plena do sistema desde agosto de 2000.

O tamanho da amostra foi estimado tendose, como referência, os diversos desfechos explorados pelo estudo. Para o cálculo da amostra, utilizou-se um poder de $80 \%$; um erro alfa de $5 \%$, para exposições variando entre $25 \%$ e $75 \%$, com uma razão de prevalência de 1,6. Ao valor obtido, foram acrescidos $10 \%$ para manejo de perdas e recusas e $15 \%$ para controle de fatores de confusão. Assim, a amostra necessária para o estudo totalizou 1.800 indivíduos.

A inclusão dos indivíduos foi realizada através de visitas domiciliares em quarenta setores censitários selecionados aleatoriamente. Em cada setor, foram sorteados trinta domicílios, totalizando 1.200 famílias, onde se esperava encontrar, em média, 1,5 pessoa/casa na faixa etária em estudo 15. A partir do quarteirão previamente sorteado, escolheu-se, também aleatoriamente, o domicílio de início das entrevistas. Ao terminar as entrevistas de um determinado domicílio, duas casas eram evitadas antes de a próxima residência ser visitada.

Entre as 2.177 pessoas encontradas, foram entrevistadas 1.968 pessoas. Dessa forma, as perdas e recusas individuais atingiram 9,6\%. A distribuição da amostra, em relação a sexo e idade, foi semelhante àquela encontrada no recenseamento para a zona urbana de Pelotas. Foram incluídos, na análise, os indivíduos que auto-referiram hipertensão arterial sistêmica.

A coleta de dados foi realizada através da aplicação de questionários padronizados e précodificados. Os entrevistadores desconheciam os objetivos do estudo e foram submetidos a um programa de treinamento para padronização da coleta, incluindo medidas de peso e altura. Durante as atividades de treinamento, foi realizado estudo piloto.

O manejo clínico da hipertensão foi classificado como adequado quando preenchia os três critérios: realização de, no mínimo, duas consultas médicas no último ano que antecedeu a data da entrevista; realização dos exames complementares (eletrocardiograma, exame de urina e exame de sangue) desde que foi comunicado o diagnóstico da doença; e recebimento de recomendações médicas necessárias para o controle dos níveis de pressão dos entrevistados hipertensos indicadas de acordo com as características de vida individuais (redução da ingestão de sal, orientação nutricional para redução de peso, redução da ingestão de gordura, cessar consumo de bebidas alcoólicas, parar de fumar e praticar exercícios físi$\cos )$. O não preenchimento de quaisquer dos critérios acima foi suficiente para classificar o manejo da hipertensão arterial como inadequado. A presença das recomendações médicas foi avaliada somente quando o entrevistado referiu apresentar algum hábito comportamental relacionado com determinados fatores de risco para as doenças do aparelho circulatório incluídos no estudo. Dessa forma, os indivíduos que não receberam a recomendação porque não apresentavam o hábito comportamental relacionado ingressaram na categoria de recomendação adequada. Assim, por exemplo, os indivíduos que não fumavam foram classificados como tendo recebido a recomendação adequada para parar de fumar.

Foi realizada análise com o intuito de identificar as características dos indivíduos que receberam manejo inadequado. Foram incluídas variáveis independentes referentes às características demográficas, sócio-econômicas, comportamentais, morbidades associadas e utilização dos serviços de saúde. Todas as variáveis incluídas no modelo estavam associadas ao acesso e à qualidade dos cuidados em saúde $16,17,18$. Por exemplo, ainda que embora não tenham sido encontradas evidências definitivas da associação entre depressão e hipertensão 
arterial, sabe-se que a presença de distúrbios psiquiátricos menores pode comprometer o manejo e o prognóstico da hipertensão 19.

As variáveis demográficas incluídas no estudo foram: sexo (masculino e feminino), idade (categorizada em grupos de dez anos), estado civil (casado, em união, viúvo, separado e solteiro) e cor da pele observada pelo entrevistador (branca e não branca).

Entre as variáveis sócio-econômicas foram incluídas: escolaridade (analfabeto/primário incompleto; primário completo/ginasial incompleto; ginasial completo/colegial incompleto; colegial completo/superior incompleto; e superior completo), classificação econômica 20 e renda familiar em salários mínimos (menor que 1,01 salário; entre 1,01 e 3,0 salários; entre 3,01 e 6,0 salários; entre 6,01 e 10,0 salários; e maior que 10,0 salários).

As variáveis comportamentais que incluíam alguns fatores de risco para doenças do aparelho circulatório: consumo de álcool, vício de fumar, ingestão de sal adicional aos alimentos já preparados, nível de atividade física no lazer e classificação do estado nutricional.

O consumo de álcool foi medido a partir de questões relativas à freqüência, ao tipo e à quantidade da ingestão de bebidas alcoólicas. O ponto de corte para definir consumo abusivo de álcool foi $30 \mathrm{~g} /$ dia de etanol ou mais 21 .

$\mathrm{O}$ vício de fumar foi analisado em duas categorias: fumantes e não fumantes; a ingestão de sal adicional aos alimentos preparados foi coletada de forma dicotômica.

O nível de atividade física foi estabelecido a partir da quantidade, duração e tipo de esforço físico desempenhado nas atividades de lazer, classificando-se os indivíduos em suficientemente ativos e insuficientemente ativos 22,23.

As morbidades incluídas no estudo foram: sobrepeso ou obesidade, diabetes mellitus referido e presença de distúrbios psiquiátricos menores.

Através das medidas de peso e altura, os indivíduos foram classificados quanto ao estado nutricional segundo o Índice de Massa Corporal (IMC). O ponto de corte para definir sobrepeso foi IMC $\geq 25 \mathrm{~kg} / \mathrm{m}^{2} 24$. Os indivíduos com sobrepeso e obesidade constituíram uma única categoria.

Os indivíduos considerados com diabetes mellitus foram aqueles que referiram diagnóstico da doença realizado por médico.

A presença de distúrbios psiquiátricos menores foi estabelecida pela aplicação do Self Reported Questionnaire (SRQ-20), com ponto de corte de seis para homens e sete para mulheres 25 .

As variáveis relacionadas à utilização de serviços de saúde foram: local de realização das consultas médicas (sistema público, serviços contratados, serviços conveniados e seguros, e sistema privado) e se o indivíduo consultava com o mesmo médico nos últimos 12 meses desde a aplicação do questionário.

A criação do banco de dados foi realizada no programa Epi Info, versão 6.0 (Centers for Disease Control and Prevention, Atlanta, Estados Unidos), através de dupla entrada, com posterior avaliação da consistência e coerência. A análise foi realizada no programa SPSS, versão 11 (SPSS Inc., Chicago, Estados Unidos) e Stata 7.0 (Stata Corporation, College Station, Estados Unidos), descrevendo-se as características relacionadas aos cuidados da hipertensão e, posteriormente, analisados os fatores associados à inadequação do manejo dos indivíduos incluídos no estudo.

As associações entre as diversas variáveis e o desfecho foram investigadas através das razões de prevalência, com cálculo dos respectivos intervalos de confiança e análise da significância estatística através do teste de qui-quadrado.

Para controle dos fatores de confusão, foi realizada regressão de Poisson seguindo o modelo de análise hierarquizado 26 , que apresentava as variáveis demográficas e sócio-econômicas num nível distal que determinava as variáveis comportamentais num nível intermediário. O conjunto proximal de variáveis, por sua vez, incluía a presença de morbidades associadas e as de utilização de serviços de saúde (Figura 1). Foram incluídas, na regressão de Poisson, as variáveis que, na análise, apresentaram valores de significância de até $20 \%$. Em cada nível da análise multivariada, foram excluídas as variáveis com níveis de significância maiores de $5 \%$.

\section{Resultados}

Foram entrevistadas 1.968 pessoas, sendo que 380 (19,3\%; IC95\%: 17,6-21,1) indivíduos referiram apresentar hipertensão arterial sistêmica.

Entre os 380 indivíduos que referiram apresentar hipertensão arterial sistêmica, 167 (44,1\%) haviam consultado com o mesmo médico durante os 12 meses anteriores à entrevista, a média anual de consultas foi 9,0 (IC95\%: 7,2-10,8). Porém, 54 (14,2\%) indivíduos não realizaram consulta médica no ano que antecedeu a entrevista.

Entre os hábitos comportamentais apresentados pelos indivíduos hipertensos estudados, verificou-se que $23(6,1 \%)$ indivíduos faziam uso de sal adicional na alimentação, 183 (48,2\%) consumiam gordura em excesso, $145(38,2 \%)$ apresentavam IMC $\geq 25 \mathrm{~kg} / \mathrm{m}^{2}, 42(11,1 \%)$ inge- 


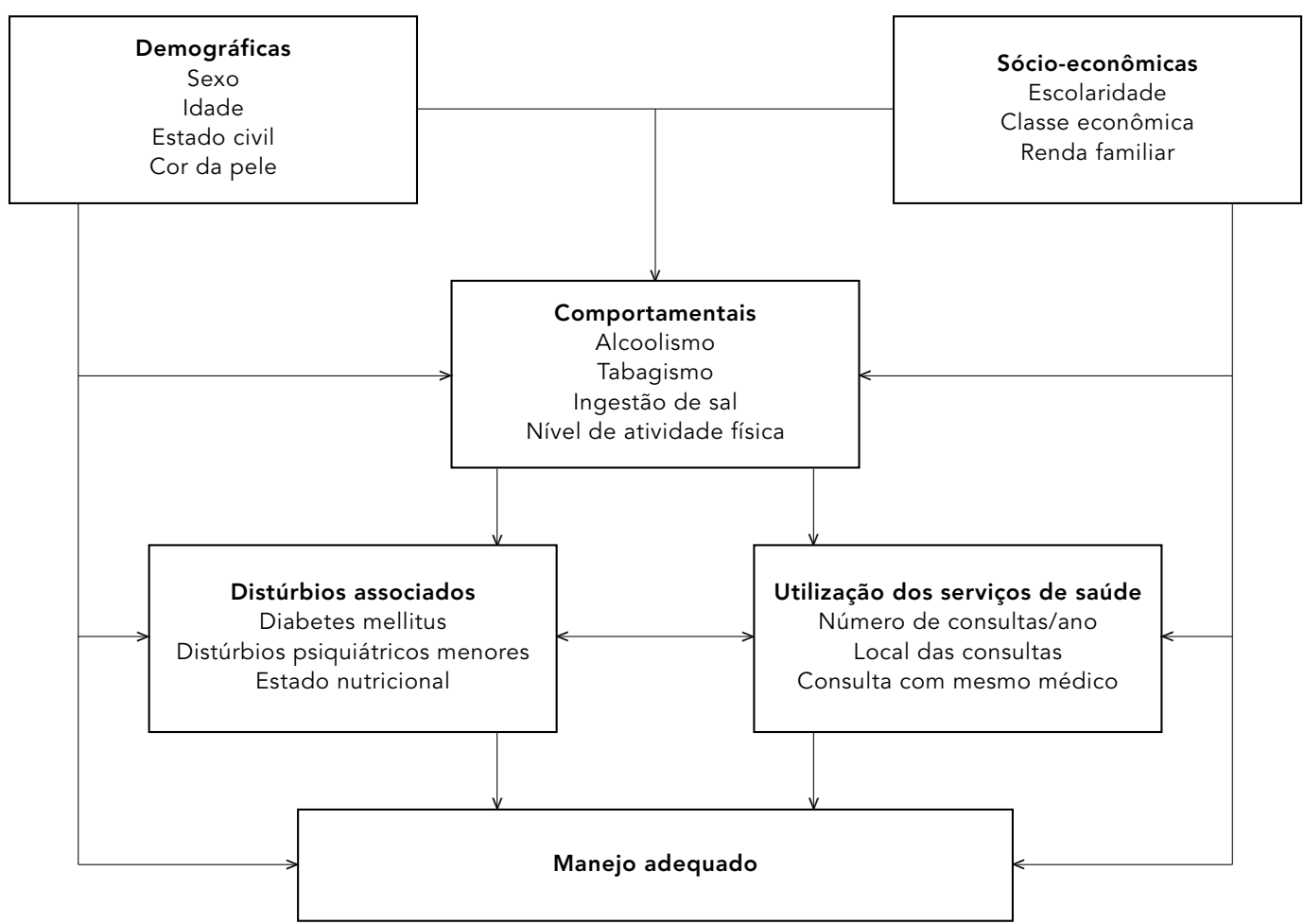

riam, no mínimo, 30g de etanol/dia, $180(47,4 \%)$ eram fumantes, e 325 (85,5\%) foram classificados como insuficientemente ativos (Tabela 1).

Em relação aos exames solicitados pelo médico, pode-se observar que os exames de sangue foram os mais requisitados $(83,7 \%)$, enquanto que o eletrocardiograma foi o menos freqüente $(74,2 \%)$ (Tabela 1$)$

Quanto às recomendações adequadas dadas pelos médicos aos hipertensos, as mais freqüentes foram reduzir a ingestão de sal $(98,4 \%)$ e interromper o consumo de bebidas alcoólicas $(93,7 \%)$; prescrever dieta para emagrecer e aconselhar comer alimentos com menos gordura atingiram, ambas, $88,2 \%$. As recomendações menos freqüentes foram parar de fumar $(73,2 \%)$ e praticar atividades físicas $(68,9 \%)$ (Tabela 1$)$.

Do total da amostra de hipertensos $(\mathrm{n}=380)$, 284 indivíduos (74,7\%; IC95\%: 70,4-79,1) referiram o manejo clínico da hipertensão arterial como inadequado (segundo os critérios descritos anteriormente).

A análise bivariada mostrou que as mulheres apresentavam probabilidade $20 \%$ menor de ma- nejo inadequado do que os homens. Os indivíduos, a partir dos cinqüenta anos, apresentaram menor prevalência de manejo inadequado do que os participantes das categorias mais jovens. Em relação ao estado civil, não foram constatadas diferenças estatisticamente significativas entre as categorias. Verificou-se que, em relação à cor da pele, os indivíduos classificados como de cor não branca referiram uma prevalência de inadequação do manejo clínico da ordem de $82 \%$, valor superior ao encontrado nos indivíduos de cor branca $(72,5 \%)$, contudo, o limite inferior do intervalo de confiança foi igual à unidade, e o teste estatístico não foi significativo (Tabela 2).

Quanto ao grau de escolaridade, observouse que os indivíduos com ensino superior completo apresentaram menor prevalência de manejo inadequado. Os indivíduos classificados nas duas categorias de escolaridade inferiores apresentaram razões de prevalência maiores quanto ao manejo clínico inadequado da hipertensão em relação aos indivíduos com maior escolaridade, entretanto as medidas de associação não foram significativas (Tabela 2). 
Os indivíduos pertencentes às classes $\mathrm{A}$ e $\mathrm{B}$ apresentaram $64,4 \%$ de inadequação do manejo clínico da hipertensão, valor inferior aos indivíduos da classe C $(75,8 \%)$ e aos das classes D e E $(80,5 \%)$, os quais apresentaram razão de prevalência $25 \%$ maior de receberem manejo inadequado em relação à categoria de base. $\mathrm{O}$ teste de tendência linear foi significativo revelando que, a cada mudança para categorias de classificação econômica inferiores, o percentual de indivíduos referindo manejo inadequado da hipertensão aumentava.

As pessoas com renda familiar de até um salário mínimo referiram manejo inadequado da hipertensão com maior freqüência do que aquelas de renda mais elevada. Para as demais categorias de renda, a análise não mostrou diferenças estatisticamente significativas. $O$ teste de tendência linear foi significativo, mostrando uma relação inversa entre renda e manejo inadequado da doença, quanto maior a renda, menor a inadequação (Tabela 2).

Entre as variáveis comportamentais foram encontradas diferenças estatisticamente significativas em relação ao consumo de álcool, ao tabagismo e ao manejo da hipertensão. Entre os indivíduos que consumiam, no mínimo, $30 \mathrm{~g}$ de etanol/dia, 95,2\% relataram manejo inadequado. Os indivíduos classificados nessa categoria apresentaram o maior percentual de manejo inadequado da amostra. Os intervalos de confiança e o teste estatístico confirmaram as diferenças. O teste de tendência linear revelou que, com o aumento do consumo de bebidas alcoólicas, aumentava o percentual de indivíduos com manejo inadequado. Quanto ao tabagismo, a freqüência de inadequação foi de $63,5 \%$ entre os não fumantes e $87,2 \%$ nos fumantes. Nos indivíduos suficientemente ativos, foi observada uma inadequação de manejo de $65,5 \%$, enquanto que os indivíduos insuficientemente ativos relataram $76,3 \%$ de inadequação. Entretanto, não se observou associação estatística. Quanto ao consumo de sal adicional, a análise não revelou diferença estatisticamente significativa (Tabela 3 ).

Observando-se as morbidades associadas, verificou-se que os indivíduos com diabetes mellitus apresentaram uma razão de prevalência $27 \%$ menor de serem manejados inadequadamente. Constatou-se um percentual de inadequação de 56,6\% entre os diabéticos e 77,7\% entre as pessoas sem a doença (Tabela 3 ). As análises da associação do estado nutricional dos indivíduos e da presença de distúrbios psiquiátricos menores com o manejo clínico da hipertensão arterial não revelaram significância estatística.
Tabela 1

Distribuição dos fatores de risco, de realização de alguns exames

e recomendações médicas adequadas com respectivos intervalos de confiança.

Pelotas, Rio Grande do Sul, Brasil, 2000.

\begin{tabular}{lrcc}
\hline & $\mathbf{n}$ & Prevalência & IC95\% \\
\hline Hábitos comportamentais & & & \\
Uso de sal adicional & 23 & 6,1 & $3,7-8,5$ \\
Consumo de gordura em excesso & 183 & 48,2 & $43,1-53,2$ \\
Sobrepeso ou obesidade & 145 & 38,2 & $33,3-43,0$ \\
Alcoolismo & 42 & 11,1 & $7,9-14,2$ \\
Tabagismo & 180 & 47,4 & $42,3-52,4$ \\
Sedentarismo & 325 & 85,5 & $82,0-89,1$ \\
& & & \\
Realização de exames & & & \\
ECG & 282 & 74,2 & $69,8-78,6$ \\
Exame de urina & 300 & 78,9 & $74,8-83,0$ \\
Exame de sangue & 318 & 83,7 & $80,0-87,4$ \\
& & & \\
Recomendações adequadas & & & \\
Diminuir sal da alimentação & 374 & 98,4 & $97,2-99,7$ \\
Orientação nutricional & 335 & 88,2 & $84,9-91,4$ \\
Comer menos gordura & 335 & 88,2 & $84,9-91,4$ \\
Cessar consumo de álcool & 356 & 93,7 & $91,2-96,1$ \\
Parar de fumar & 278 & 73,2 & $68,7-77,6$ \\
Praticar atividade física & 262 & 68,9 & $64,3-73,6$ \\
\hline
\end{tabular}

Em relação às variáveis de utilização de serviços de saúde, local de consulta e consultas com o mesmo médico nos últimos doze meses, não foram observadas diferenças estatisticamente significativas (Tabela 3 ).

Após a análise da regressão de Poisson, mantiveram-se associados ao desfecho as variáveis de sexo, idade, classificação econômica, hábito de tabagismo e presença de diabetes mellitus, com a mesma direção observada na análise bivariada (Tabela 4 ).

\section{Discussão}

Estudos de base populacional apresentam a vantagem de poderem representar o conjunto total de onde foi retirada a amostra 27 , constituindo-se em fonte importante de informações para planejar, administrar, organizar e avaliar sistemas ou serviços de saúde.

A partir da revisão, elaborou-se o desfecho realizando uma combinação de variáveis coletadas durante as entrevistas. Sabe-se, por exemplo, que a freqüência de acompanhamento médico para pacientes com hipertensão arterial sistêmica depende de fatores como gravidade 
Distribuição de manejo adequado em relação às variáveis demográficas e sócio-econômicas. Pelotas, Rio Grande do Sul, Brasil, 2000.

\begin{tabular}{|c|c|c|c|c|c|c|}
\hline & $\mathbf{N}$ & $\%$ & $\begin{array}{c}\text { Inadequação } \\
(\%)\end{array}$ & $\begin{array}{l}\text { Razão de } \\
\text { prevalência }\end{array}$ & IC95\% & $p$ valor \\
\hline \multicolumn{7}{|l|}{ Sexo } \\
\hline Masculino & 114 & 30,0 & 87,7 & 1,00 & & $<0,001$ \\
\hline Feminino & 266 & 70,0 & 69,2 & 0,79 & $0,71-0,88$ & \\
\hline \multicolumn{7}{|l|}{ Idade (anos) } \\
\hline $20-39$ & 66 & 17,4 & 87,9 & 1,00 & & 0,04 \\
\hline $40-49$ & 83 & 21,8 & 75,9 & 0,86 & $0,74-1,00$ & \\
\hline $50-59$ & 136 & 35,8 & 69,9 & 0,79 & $0,69-0,92$ & \\
\hline $60-69$ & 95 & 25,0 & 71,6 & 0,81 & $0,70-0,95$ & \\
\hline \multicolumn{7}{|l|}{ Estado civil } \\
\hline Casado/Em união & 244 & 64,2 & 74,2 & 1,00 & & 0,97 \\
\hline Viúvo & 54 & 14,2 & 74,1 & 1,00 & $0,84-1,19$ & \\
\hline Separado & 30 & 7,9 & 76,7 & 1,03 & $0,84-1,28$ & \\
\hline Solteiro & 52 & 13,7 & 76,9 & 1,04 & $0,88-1,22$ & \\
\hline \multicolumn{7}{|l|}{ Cor da pele } \\
\hline Branca & 291 & 76,6 & 72,5 & 1,00 & & 0,07 \\
\hline Não branca & 89 & 23,4 & 82,0 & 1,13 & $1,00-1,28$ & \\
\hline \multicolumn{7}{|l|}{ Escolaridade } \\
\hline Superior completo & 34 & 9,0 & 58,8 & 1,00 & & 0,23 \\
\hline $\begin{array}{l}\text { Colegial completo } \\
\text { e superior incompleto }\end{array}$ & 62 & 16,4 & 74,2 & 1,26 & $0,92-1,73$ & \\
\hline $\begin{array}{l}\text { Ginásio completo } \\
\text { e colegial incompleto }\end{array}$ & 63 & 16,7 & 73,0 & 1,24 & $0,90-1,71$ & \\
\hline $\begin{array}{l}\text { Primário completo } \\
\text { e ginásio incompleto }\end{array}$ & 89 & 23,5 & 75,5 & 1,32 & $0,97-1,78$ & \\
\hline $\begin{array}{l}\text { Analfabeto e primário } \\
\text { incompleto }\end{array}$ & 130 & 34,4 & 77,7 & 1,32 & $0,98-, 78$ & \\
\hline \multicolumn{7}{|l|}{ Classificação econômica } \\
\hline$A$ e $B$ & 101 & 26,7 & 64,4 & 1,00 & & $0,015^{\star}$ \\
\hline $\mathrm{C}$ & 128 & 33,9 & 75,8 & 1,18 & $0,99-1,40$ & \\
\hline$D$ e $E$ & 149 & 39,4 & 80,5 & 1,25 & $1,06-1,48$ & \\
\hline \multicolumn{7}{|l|}{$\begin{array}{l}\text { Renda familiar } \\
\text { (salários mínimos) }\end{array}$} \\
\hline$>6,01$ & 39 & 10,3 & 59,0 & 1,00 & & $0,032^{\star \star}$ \\
\hline $3,01-6,00$ & 53 & 14,1 & 75,5 & 1,28 & $0,94-1,73$ & \\
\hline $1,01-3,00$ & 155 & 41,1 & 72,3 & 1,23 & $0,93-1,62$ & \\
\hline$<1,01$ & 130 & 34,5 & 81,5 & 1,38 & $1,05-1,82$ & \\
\hline
\end{tabular}

* Teste de tendência linear $<0,01$

** Teste de tendência linear $=0,01$. 
Distribuição de manejo adequado em relação às variáveis comportamentais, morbidades associadas e utilização de serviços de saúde. Pelotas, Rio Grande do Sul, Brasil, 2000.

\begin{tabular}{|c|c|c|c|c|c|c|}
\hline Variável & $\mathbf{N}$ & $\%$ & $\begin{array}{l}\text { Adequação } \\
(\%)\end{array}$ & $\begin{array}{l}\text { Razão de } \\
\text { prevalência }\end{array}$ & $\mathrm{IC} 95 \%$ & $p$ valor \\
\hline \multicolumn{7}{|l|}{ Consumo de álcool } \\
\hline Não bebe & 122 & 32,1 & 68,9 & 1,00 & & $0,003^{*}$ \\
\hline Menos de 30g/dia & 216 & 56,8 & 74,1 & 1,08 & $0,93-1,24$ & \\
\hline Igual ou + de 30g/dia & 42 & 11,1 & 95,2 & 1,38 & $1,21-1,59$ & \\
\hline \multicolumn{7}{|l|}{ Tabagismo } \\
\hline Não & 200 & 52,6 & 63,5 & 1,00 & & $<0,001$ \\
\hline Sim & 180 & 47,4 & 87,2 & 1,37 & $1,22-1,55$ & \\
\hline \multicolumn{7}{|l|}{ Ingestão de sal adicional } \\
\hline Não & 357 & 93,9 & 75,1 & 1,00 & & 0,556 \\
\hline Sim & 23 & 6,1 & 69,6 & 0,93 & $0,70-1,22$ & \\
\hline \multicolumn{7}{|l|}{ Nível de atividade física } \\
\hline Suficientemente ativo & 55 & 14,5 & 65,5 & 1,00 & & 0,087 \\
\hline Insuficientemente ativo & 325 & 85,5 & 76,3 & 1,17 & $0,95-1,43$ & \\
\hline \multicolumn{7}{|l|}{ Estado nutricional } \\
\hline Normal & 93 & 24,6 & 76,3 & 1,00 & & 0,657 \\
\hline Sobrepeso e obeso & 285 & 75,4 & 74,0 & 0,97 & $0,85-1,11$ & \\
\hline \multicolumn{7}{|l|}{ Diabetes mellitus } \\
\hline Não & 327 & 86,1 & 77,7 & 1,00 & & 0,001 \\
\hline Sim & 53 & 13,9 & 56,6 & 0,73 & $0,57-0,93$ & \\
\hline \multicolumn{7}{|l|}{$\begin{array}{l}\text { Distúrbios psiquiátricos } \\
\text { menores }\end{array}$} \\
\hline Não & 222 & 58,6 & 72,5 & 1,00 & & 0,253 \\
\hline $\operatorname{Sim}$ & 157 & 41,4 & 77,7 & 1,07 & $0,95-1,20$ & \\
\hline \multicolumn{7}{|l|}{ Local de consulta } \\
\hline Sistema privado & 49 & 22,2 & 59,2 & 1,00 & & 0,536 \\
\hline Convênios/Seguros saúde & 52 & 23,5 & 67,7 & 1,14 & $0,84-1,54$ & \\
\hline Serviços contratados & 21 & 9,5 & 61,9 & 1,05 & $0,70-1,57$ & \\
\hline Sistema público & 99 & 44,8 & 70,7 & 1,19 & $0,92-1,56$ & \\
\hline \multicolumn{7}{|l|}{$\begin{array}{l}\text { Consulta com o mesmo } \\
\text { médico }\end{array}$} \\
\hline Sim & 167 & 75,6 & 64,7 & 1,00 & & 0,307 \\
\hline Não & 54 & 24,4 & 72,2 & 1,12 & $0,91-1,36$ & \\
\hline
\end{tabular}

* Teste de tendência linear $<0,01$. 
Tabela 4

Análise multivariada, regressão de Poisson, inadequação do tratamento para hipertensão arterial sistêmica. Pelotas, Rio Grande do Sul, Brasil, 2000.

\begin{tabular}{|c|c|c|c|}
\hline Variável & $\begin{array}{l}\text { Razão de } \\
\text { prevalência }\end{array}$ & IC95\% & p valor \\
\hline \multicolumn{4}{|l|}{ Sexo* } \\
\hline Masculino & 1,00 & & \\
\hline Feminino & 0,76 & $0,68-0,85$ & $<0,001$ \\
\hline \multicolumn{4}{|l|}{ Idade (anos)* } \\
\hline $20-39$ & 1,00 & & \\
\hline $40-49$ & 0,91 & $0,79-1,05$ & 0,20 \\
\hline $50-59$ & 0,83 & $0,72-0,96$ & 0,01 \\
\hline $60-69$ & 0,83 & $0,71-0,97$ & 0,02 \\
\hline \multicolumn{4}{|l|}{ Raça* } \\
\hline Branca & 1,00 & & \\
\hline Não branca & 1,07 & $0,95-1,20$ & 0,30 \\
\hline \multicolumn{4}{|l|}{ Classificação econômica* } \\
\hline$A$ e $B$ & 1,00 & & \\
\hline C & 1,21 & $1,01-1,44$ & 0,04 \\
\hline$D$ e $E$ & 1,27 & $1,08-1,50$ & $<0,01$ \\
\hline \multicolumn{4}{|l|}{ Tabagismo** } \\
\hline Não & 1,00 & & \\
\hline Sim & 1,28 & $1,13-1,46$ & $<0,001$ \\
\hline \multicolumn{4}{|l|}{ Consumo de álcool ${ }^{\star \star}$} \\
\hline Não bebe & 1,00 & & \\
\hline Menos de 30g/dia & 1,02 & $0,89-1,17$ & 0,79 \\
\hline Igual ou + de $30 \mathrm{~g} / \mathrm{dia}$ & 1,11 & $0,95-1,30$ & 0,19 \\
\hline \multicolumn{4}{|l|}{ Nível de atividade física** } \\
\hline Suficientemente ativo & 1,00 & & \\
\hline Insuficientemente ativo & 1,17 & $0,97-1,41$ & 0,09 \\
\hline \multicolumn{4}{|l|}{ Diabetes referido*** } \\
\hline Não & 1,00 & & \\
\hline Sim & 0,73 & $0,58-0,93$ & 0,01 \\
\hline
\end{tabular}

* Variáveis que ingressaram no primeiro nível;

** Variáveis que ingressaram no segundo nível

*** Variáveis que ingressaram no terceiro nível. ma de repouso 9,29,30. No instrumento de entrevista, conseguiu-se especificar exame de urina e eletrocardiograma. Entretanto, considerou-se pouco provável que os respondentes soubessem especificar a natureza dos exames séricos. Assim, a resposta positiva para realização de "exame de sangue" foi considerada como suficiente para o conjunto das provas séricas.

As recomendações realizadas pelos médicos incluídas no desfecho são as que constam nas diretrizes nacionais e internacionais para $o$ manejo da hipertensão arterial sistêmica 9,30,31 .

Entre os indivíduos hipertensos incluídos no estudo, constatou-se um padrão de utilização anual de serviços de saúde bem superior àquele encontrado para a população adulta de Pelotas, pois, em outra investigação de corte transversal, foi encontrada uma média anual de três consultas 32. Contudo, verificou-se que mais da metade das pessoas $(55,9 \%)$ não consultaram com o mesmo médico, o que poderia demonstrar a falta de vínculo entre médicos e pacientes. Outro fator que poderia justificar o achado é o fato de que a maior parte da amostra constitui-se de pacientes atendidos pelo SUS, que, certamente, não têm a mesma opção de decidir consultar com o mesmo médico em relação aos indivíduos atendidos por convênios e na rede privada.

Em Pelotas, a partir de dados coletados em 1992, foi realizado um estudo que descrevia e analisava o manejo de pacientes com hipertensão arterial sistêmica ${ }^{33}$. O estudo descreveu a prevalência de alguns hábitos comportamentais utilizados na presente investigação, sendo que consumo de sal, tabagismo e estado nutricional foram coletados da mesma forma. Observou-se que, no transcorrer de quase dez anos, o consumo de sal entre a população de hipertensos manteve-se semelhante $(5 \%$ em $1992 \mathrm{e}$ $6,1 \%$ em 2000), o tabagismo aumentou (28\% em 1992 e 47,4\% em 2000) e a prevalência de obesidade diminuiu (55\% em 1992 e 38,2\% em 2000). Os achados do presente estudo, nos indivíduos selecionados, contrariam as tendências observadas atualmente em outros estudos que mostram uma redução no hábito de fumar e aumento da obesidade 34,35 . Deve-se ressaltar que o estudo anterior selecionou os indivíduos a partir de medidas de pressão e pela utilização de medicação anti-hipertensiva, e este, pela autoreferência, podendo justificar essa diferença.

Em relação à solicitação de exames, os percentuais entre os dois estudos realizados em Pelotas não mostraram alterações. Em 1992, os testes sanguíneos foram referidos por $84 \%$ dos indivíduos, e a realização de eletrocardiograma foi relatada por $74 \%$ dos pacientes 33 . Porém, 
quanto às recomendações realizadas pelos médicos, foram observadas diferenças entre as duas investigações. Os percentuais observados no presente estudo, sugerindo a redução do consumo de sal adicional, a diminuição da ingestão de gorduras, a perda de peso, a prática de exercícios físicos e cessação do vício de fumar, todos, foram superiores aos encontrados em Pelotas na outra investigação. Esse achado sugere uma melhor compreensão do valor das medidas não medicamentosas envolvidas no manejo dos pacientes com hipertensão arterial sistêmica $31,34,36,37$.

Os resultados do presente estudo mostram que apenas 1/4 dos pacientes foi manejado adequadamente. A análise ajustada para os diversos fatores de confusão revelou achados positivos e negativos em relação à qualidade dos cuidados. As mulheres, as pessoas com mais de cinqüenta anos e os indivíduos que apresentavam diabetes mellitus tiveram um maior percentual de cuidados adequados. Esse achado reflete certo nível de qualificação do manejo, pois se sabe que a hipertensão arterial sistêmi-

\section{Resumo}

Foi realizado um estudo transversal com base populacional entre os indivíduos de 20 a 69 anos, residentes na zona urbana de Pelotas, Rio Grande do Sul, Brasil. O objetivo foi avaliar o manejo da hipertensão e descrever as características dos indivíduos classificados com cuidados inadequados. Entre os 1.968 entrevistados, 380 eram hipertensos (19,3\%), 44,1\% consultaram sempre com o mesmo médico. Entre os hipertensos, 85,5\% eram sedentários, 48,2\% consumiam gordura em excesso $e$ 47,4\% eram fumantes. As recomendações adequadas mais realizadas pelos médicos foram restringir o sal (98,4\%), interromper o consumo de bebidas alcoólicas (93,7\%), aconselhar dieta para emagrecer e reduzir a ingestão de gorduras (88,2\%), parar de fumar $(73,2 \%) e$ praticar atividades físicas (68,9\%). O manejo da hipertensão foi classificado como inadequado em 284 indivíduos (74,7\%; IC95\%: 70,4-79,1). Constatou-se maior probabilidade de manejo adequado nas mulheres, nos indivíduos a partir dos cinqüenta anos, nas pessoas pertencentes à classe econômica A e B, em não fumantes, e nos indivíduos com diabetes mellitus. Alguns achados traduziram aspectos positivos da atenção, contudo as diferenças de cuidados de acordo com a classe social evidenciaram a falta de eqüidade assistencial.

Hipertensão; Fatores de Risco; Avaliação de Processo (Cuidados de Saúde); Qualidade dos Cuidados de Saúde ca apresenta mais complicações clínicas e é mais grave nas pessoas mais idosas e com doenças crônicas associadas, tais como diabetes mellitus 29,36.

Por outro lado, os resultados deste estudo mostraram diferenciais na qualidade dos cuidados de saúde de acordo com as variáveis que representavam as características sócio-econômicas. As pessoas inseridas nas classes econômicas mais baixas, com menor escolaridade e com menor renda familiar apresentaram menor probabilidade de receberem manejo adequado. Esse achado foi considerado como negativo, uma vez que representa uma iniqüidade na assistência, confirmando, mais uma vez, o enunciado de Hart sobre a lei dos cuidados inversos em saúde 38. Outros estudos realizados em Pelotas já chamaram a atenção sobre diferenciais na atenção à saúde em relação às mulheres 39 , gestantes 40 e crianças ${ }^{41}$. Assim, o presente estudo incluiu os adultos de ambos os sexos em condições socialmente vulneráveis no rol das pessoas que recebem assistência inadequada.

\section{Colaboradores}

G. Sturmer, M. T. A. Olinto e A. M. B. Menezes participaram da análise e redação do artigo. J. S. Dias-daCosta foi o supervisor do trabalho de campo, participou da análise e redação do artigo. D. P. Gigante e S. Macedo participaram da redação do artigo.

\section{Agradecimentos}

Projeto de pesquisa financiado pela Fundação de Amparo à Pesquisa do Estado do Rio Grande do Sul e pelo Conselho Nacional de Desenvolvimento Científico e Tecnológico (processos: 520884/99 e 520251/ 99-7). 


\section{Referências}

1. Ministério da Saúde. Informações de saúde 2002. http:/ / www.datasus.gov.br (acessado em 27/Set/ 2005).

2. Núcleo de Informações em Saúde, Secretaria da Saúde do Rio Grande do Sul. Estatísticas de saúde: mortalidade 2002. Porto Alegre: Secretaria da Saúde do Rio Grande do Sul; 2003.

3. Lessa I. Epidemiologia da hipertensão arterial sistêmica e da insuficiência cardíaca no Brasil. Rev Bras Hipertens 2001; 8:383-92.

4. Trindade IS, Heineck G, Machado JR, Ayzemberg H, Formighieri M, Crestani M, et al. Prevalência de hipertensão arterial sistêmica na população urbana de Passo Fundo (RS). Arq Bras Cardiol 1998; 71:127-30.

5. Piccini RX, Victora CG. Hipertensão arterial sistêmica no Sul do Brasil: prevalência e fatores de risco. Rev Saúde Pública 1994; 28:261-7.

6. Fuchs FD, Moreira LB, Moraes RS, Bredemeier M, Cardozo CS. Prevalência de hipertensão arterial sistêmica e fatores de risco associados na região urbana de Porto Alegre. Estudo de base populacional. Arq Bras Cardiol 1994; 63:473-9.

7. Duncan BB, Schmidt MI, Polanczyk CA, Homrich CS, Rosa RS, Achutti AC. Fatores de risco para doenças não-transmissíveis em área metropolitana na região Sul do Brasil. Prevalência e simultaneidade. Rev Saúde Pública 1993; 27:143-8.

8. Gus I, Harzheim E, Zaslavsky C, Medina C, Gus M. Prevalência, reconhecimento e controle da hipertensão arterial sistêmica no Estado do Rio Grande do Sul. Arq Bras Cardiol 2004; 83:424-8.

9. Chobanian AV, Bakris GL, Black HR, Cushman WC, Green LA, Izzo Jr. JL, et al. The Seventh Report of the Joint National Committee on Prevention, Detection, Evaluation, and Treatment of High Blood Pressure: the JNC 7 report. JAMA 2003; 289:2560-72.

10. Hartz MA. Institucionalizar e qualificar a avaliação: outros desafios para a atenção básica. Ciênc Saúde Coletiva 2002; 7:419-21.

11. Felisberto E. Monitoramento e avaliação na atenção básica: novos horizontes. Rev Bras Saúde Matern Infant 2004; 4:317-21.

12. Santos IS, Victora CG. Serviços de saúde: epidemiologia, pesquisa e avaliação. Cad Saúde Pública 2004; 20 Suppl 2:337-41.

13. AbouZahr C, Wardlaw T. Maternal mortality at the end of a decade: signs of progress? Bul World Health Organ 2001; 79:561-8.

14. Brook RH, McGlynn EA, Cleary PD. Measuring quality of care. N Engl J Med 1996; 335 (13 Part 2):966-70.

15. Instituto Brasileiro de Geografia e Estatística. Estimativas da população. http://www.ibge.gov.br (acessado em 30/Mar/2004).

16. Mendoza-Sassi R, Béria JU. Utilización de los servicios de salud: una revisión sistemática sobre los factores relacionados. Cad Saúde Pública 2001; 17:819-32.

17. Travassos C, Viacava F, Pinheiro R, Brito A. Utilização dos serviços de saúde no Brasil: gênero, características familiares e condição social. Rev Panam Salud Publica 2002; 11:365-73.
18. Caper P. The microanatomy of health care. Health Aff 1993; 12:174-7.

19. Scalco AZ, Scalco MZ, Azul JBS, Lotufo Neto F. Hypertension and depression. Clinics 2005; 60: 241-50.

20. Associação Nacional de Empresas de Pesquisa. Critério de classificação econômica Brasil. http:// www.anep.org.br (acessado em 13/Dez/2003).

21. Moreira LB, Fuchs FD, Moraes RS, Brademeier M, Cardozo S, Fuchs SC, et al. Alcoholic beverage consumption and associated factors in Porto Alegre, a Southern Brazilian city: a population-based survey. J Studies Alcohol 1996; 57:253-9.

22. Ainsworth BE, Haskell WL, Whitt MC, Irwin ML, Swartz AM, Strath SJ, et al. Compedium of physical activities: an update of activity codes and MET intensities. Med Sci Sports Exerc 2000; 32:S498516.

23. U.S. Department of Health and Human Services. Physical activity and health: a report from the surgeon general. Atlanta: National Center for Chronic Disease Prevention and Health Promotion; 1996.

24. World Health Organization. Obesity: preventing and managing the global epidemic. Geneva: World Health Organization; 1998.

25. Mari JJ, Williams P. A validity study of a psychiatric screening questionnaire (SRQ 20) in primary care in the city of São Paulo. Br J Psychiatry 1986;148:23-6.

26. Victora CG, Huttly SR, Fuchs SC, Olinto MTA. The role of conceptual frameworks in epidemiological analysis: a hierarchical approach. Int J Epidemiol 1997; 26:224-47.

27. Abramson JH. Cross-sectional studies. In: Detels R, Holand WW, McEwen J. Omenn GS, editors. Oxford textbook of public health. New York: Oxford University Press; 1997. p. 517-35.

28. Ramsay LE, Williams B, Jonhston GD, MacGregor GA, Poston L, Potter JF, et al. British Hypertension Society guidelines for hypertension management 1999: summary. BMJ 1999; 349:630-5.

29. Fuchs FD. Hipertensão arterial sistêmica. In: Duncan B, Schimidt MI, Giugliani E, organizadores. Medicina ambulatorial: condutas de atenção primária baseadas em evidências. Porto Alegre: Artmed; 2004. p. 641-55.

30. Williams B, Poultier NR, Brown MJ, Davis M, McInnes GT, Potter JF, et al. British Hypertension Society guidelines. Guidelines for management of hypertension: report of the fourth working party of the British Hypertension Society, 2004-BHS IV. J Hum Hypertens 2004; 18:139-85.

31. Sociedade Brasileira de Cardiologia. IV diretrizes brasileiras de hipertensão arterial. Arq Bras Cardiol 2004; 82 Suppl 4:1-14.

32. Dias-da-Costa JS, Facchini LA. Utilização de serviços ambulatoriais em Pelotas: onde a população consulta? Com que freqüência? Rev Saúde Pública 1997; 31:360-9.

33. Piccini RX, Victora CG. How well is hypertension managed in the community? A population-based survey in a Brazilian city. Cad Saúde Pública 1997; 13:595-600. 
34. Jacoby E. The obesity epidemic in the Americas: making healthy choices the easiest choices. Rev Panam Salud Publica 2004; 15:278-84.

35. Menezes AM, Jardim JR, Perez-Padilla R, Camelier A, Rosa F, Nascimento O, et al. Prevalence of chronic obstructive pulmonary disease and associated factors: the PLATINO Study in São Paulo, Brazil. Cad Saúde Pública 2005; 21:1565-73.

36. British Cardiac Society/British Hiperlipidaemia Association/British Hypertension Society/British Diabetic Association. Joint British recommendations on prevention of coronary heart disease in clinical practice: summary. BMJ 2000; 320:705-8.

37. Sacks DB, Bruns DE, Goldstein DE, Maclaren NK, McDonald JM, Parrot M. Guidelines and recommendations for laboratory analysis in the diagnosis and management of diabetes mellitus. Clin Chem 2002; 48:436-72.
38. Hart JT. The inverse care law. Lancet 1971; 1:405-12.

39. Quadros CAT, Victora CG, Dias-da-Costa JS. Coverage and focus of a cervical cancer prevention program in southern Brazil. Rev Panam Salud Publica 2004; 16:223-32.

40. Dias-da-Costa JS, Victora CG, Barros FC, Halpern R, Horta BL, Manzolli P. Assistência médica materno-infantil em duas coortes de base populacional no Sul do Brasil: tendências e diferenciais. Cad Saúde Pública 1996; 12 Suppl 1:59-66.

41. Victora CG, Vaughan JP, Barros F, Silva AC, Tomasi E. Explaining trends in inequities: evidence from Brazilian child health studies. Lancet 2000; 356 : 1093-8.

Recebido em 25/Nov/2005

Versão final reapresentada em 07/Mar/2006

Aprovado em 13/Mar/2006 